\title{
Use of Auto-titrating Positive Airway Pressure Devices for Sleep- disordered Breathing: The Good, the Bad and the Ugly
}

Gaurav Nigam ${ }^{1 *}$, Muhammad Riaz ${ }^{2}$, Charu Pathak ${ }^{3}$, Nikita Malaiya ${ }^{4}$ and Anita Valanju Shelgikar ${ }^{5}$

${ }^{1}$ Clay County Hospital, 911 Stacy Burk Drive Flora, IL 62839, USA

${ }^{2}$ Twin Cities Community Hospital, 1100 Las Tablas Rd, Templeton, CA 93465, USA

${ }^{3}$ Government Medical College, Jabalpur, India

${ }^{4}$ Sri Aurobindo Institute of Medical Sciences, Indore, India

${ }^{5}$ Sleep Disorders Center, University of Michigan, 1500 E Medical Center Dr, Ann Arbor, MI 48109, USA

\begin{abstract}
Auto-titrating positive airway pressure (APAP) devices are an effective treatment alternative for certain patients with obstructive sleep apnea (OSA). In patients with positional sleep apnea and sleep stage dependent OSA, APAP devices may be more preferable than continuous positive airway pressure (CPAP). The myriad of APAP machines currently available employ increasingly sophisticated mathematical algorithms to identify and ameliorate respiratory disturbances. The cardiovascular benefits accrued from consistent CPAP usage still remain to be proven with APAP use. Although a reasonable alternative to CPAP, APAP titration or treatment should not be used in patients in whom OSA coexists with risk factors for central sleep apnea and hypoventilation syndromes.
\end{abstract}

Keywords: APAP; CPAP; Adherence; Sleep disordered breathing; Obstructive sleep apnea; Apnea hypopnea index; Treatment emergent central sleep apnea

\section{Introduction}

Obstructive sleep apnea (OSA) is a major public health problem in the United States, with the economic burden of disease incurring annual costs higher than that of asthma or chronic obstructive pulmonary disease and fairly close to that of diabetes mellitus [1]. An 18-year mortality follow-up study conducted on the populationbased Wisconsin Sleep Cohort sample found a high mortality risk with untreated sleep-disordered breathing, independent of age, sex, and body mass index [2]. This highlights the importance of timely diagnosis and treatment of obstructive sleep apnea. Attended polysomnography (PSG) and unattended home sleep apnea testing or HSAT (also known as out of center sleep testing or cardiorespiratory polygraphy) are the two modalities commonly used to diagnose OSA. Studies with similar results between diagnostic PSG and HSAT have mainly been performed in selected patients with high pre-test probability of OSA [3]. Utilization of HSAT for the diagnosis of OSA should be limited to select patients with a high pretest probability for OSA.

Unattended auto-titrating positive airway pressure (APAP) titration appears to be a simple, cost effective method for treatment of OSA [4]. When compared to attended titration using continuous positive airway pressure (CPAP), APAP expedites treatment of OSA after the diagnosis has been made using attended PSG or unattended HSAT. Given these advantages, APAP is increasingly used to treat OSA. Here we review the literature to understand the benefits and pitfalls of using APAP to treat obstructive sleep apnea.

\section{The good}

There are certain patients for whom APAP is a suitable treatment option. These include patients with prominent sleep stage and body position dependent worsening of OSA and those who anticipate rapid weight fluctuations [5]. Positional sleep apnea is found to be present in $7-50 \%$ of patients with obstructive sleep apnea, depending on severity of baseline OSA [6]. In these subgroups APAP appears to be more effective than CPAP, at least during the initial course of treatment [5]. Pressure requirements change considerably in bariatric surgery patients undergoing rapid weight loss, and APAP devices are promising in management of positive airway pressure therapy during this time [7].
APAP devices could be considered a reasonable option for patients in whom insomnia is a major cause, component or consequence of obstructive sleep apnea. Auto-titrating devices improved the sleep efficiency and modulated the sleep architecture to enhance the percentage of total sleep time in slow wave sleep, in some studies comparing APAP to CPAP therapy $[8,9]$. One study found that insomnia related to OSA symptoms improved with APAP treatment, with maximal improvement reported in patients with higher severity of OSA and excessive daytime somnolence [10]. APAP may be specifically suited for certain elderly patients because of the preponderance of lighter stages sleep [11] and prolonged obstructive apneas in the elderly compared to young adults [12]. Konermann et al. have demonstrated that compared to CPAP, auto-titrating devices decrease the mean duration of apneas, decrease the arousal index and increase the amount of slow wave sleep, all of which would lead to consolidated sleep in the elderly [8]. This could be related to APAP devices delivering lower average pressures when compared to conventional CPAP machines.

APAP might be a preferred treatment modality for obstructive sleep apnea during pregnancy for certain patients. Hormonal and weight changes during pregnancy can alter the severity of sleep-disordered breathing over the period of gestation. In one study, 50\% of pregnant subjects with obstructive sleep apnea required increase in CPAP setting at a 24-week gestation re-titration study, compared to their first trimester titration study. The increased pressure requirement was seen mostly in patients with substantial weight gain during pregnancy [13].

Clinical pearl: Auto-titrating positive airway pressure devices appear to be a promising treatment option in patients with positional sleep apnea, sleep stage dependent obstructive sleep apnea, rapid body weight

${ }^{*}$ Corresponding author: Gaurav Nigam, MD, Clay County Hospital, 911 Stacy Burk Drive Flora, IL 62839, USA, Tel: 618662131; Fax: 6186629387; E-mail: dr.nigam@claycountyhospital.org

Received March 28, 2016; Accepted April 25, 2016; Published April 27, 2016

Citation: Nigam G, Riaz M, Pathak C, Malaiya N, Shelgikar AV (2016) Use of Auto-titrating Positive Airway Pressure Devices for Sleep-disordered Breathing: The Good, the Bad and the Ugly. J Pulm Respir Med 6: 336. doi:10.4172/2161105X.1000336

Copyright: ( 2016 Nigam G, et al. This is an open-access article distributed under the terms of the Creative Commons Attribution License, which permits unrestricted use, distribution, and reproduction in any medium, provided the original author and source are credited. 
fluctuations, and in those whose obstructive sleep apnea is associated with sleep fragmentation and insomnia. Long term studies comparing efficacy of CPAP to APAP are needed to identify which pregnant patients might be best served by an auto-titrating device.

Auto-titrating devices provide an option for expedited treatment in patients who have been diagnosed with OSA but have yet not undergone a CPAP titration study. This is especially pertinent given that attended CPAP titration studies can be associated with higher cost and longer wait times [4]. Also, patient reluctance or inability to sleep overnight in a sleep laboratory may preclude the ability to obtain an attended titration study. Due to the aforementioned reasons, attended in-laboratory CPAP titration studies are increasingly being supplanted by unattended at-home APAP titrations. Titration success rates (defined as mitigation of residual AHI to under 10 events per hour at the most effective tested setting) have been found to be similar when attended CPAP titrations are compared to at home APAP titration studies [14]. Also, nightly home use of APAP for a few weeks to identify the most effective pressure, followed by transition to fixed CPAP based on the data from APAP use, has similar acceptance and adherence when compared to the pressure setting obtained via single night attended manual CPAP titration [14].

A new emerging pragmatic approach is an in laboratory, attended APAP titration study instead of conventional in-laboratory, attended CPAP titration study. When APAP titrations are conducted in a sleep laboratory, a sleep technologist might be able to oversee a greater number of studies compared to what he/she could do when working on CPAP titrations [15]. This is because the sleep technologist is not expending time and effort to identify and uptitrate for apneas, hypopneas, snoring and flow limitation when working with APAP devices. While the inherent algorithm of an APAP device is working to attain the most effective pressure setting for a given patient, the technologist can pay attention to other nuances of a titration such as mask leaks, supplemental oxygen requirement, potential abnormalities on electrocardiogram or electroencephalogram, and need for wedge pillow or positional therapy to resolve obstructive events. Such events would have been possibly missed in an unattended, home APAP titration study or situations where titration studies are altogether skipped and APAP is prescribed empirically to patients diagnosed with OSA.

Clinical pearl: APAP titration done at a patient's home may offer a therapeutic pressure very similar to that obtained by a single night attended manual CPAP titration study. For select patients with OSA, home-based APAP titration studies may obviate the need for attended CPAP titration without compromising the eventual treatment recommendation.

Long term adherence to APAP seems to be good. One study showed that $75 \%$ patients adhered to APAP treatment over five years [16]. Baseline apnea-hypopnea index (AHI) is a major determinant of adherence to APAP therapy. In one study patients with AHI $>60$ events/ hour had better adherence than those below 60 events/hour [17]. While some studies [18] failed to show any difference in relative adherence to APAP versus CPAP, other studies $[9,19,20]$ showed that APAP increases "machine on" time by 10-15 minutes per night compared with conventional CPAP. APAP delivers lower pressures and results in lower pressure leaks and fewer reported side effects $[9,18,21]$. This, in turn, may improve sleep architecture, which may explain the slight difference in adherence to therapy with APAP use. One study found that more patients requiring higher fixed pressure $\left(\geq 8 \mathrm{~cm} \mathrm{H}_{2} \mathrm{O}\right)$ preferred APAP, whereas those requiring lower pressure $\left(<8 \mathrm{~cm} \mathrm{H}_{2} \mathrm{O}\right)$ preferred CPAP
[22]. This could be due to APAP being sensitive and responsive to favorable body position (e.g. non-supine) and sleep stage (e.g. slow wave sleep) leading to use of the lowest effective pressure that maintains upper airway patency. CPAP, on the other hand, would continue to keep the pressure empirically high. Amongst subjects reporting side effects with use of positive airway pressure therapy, APAP users tend to have better adherence compared to conventional CPAP users [21]. The polysomnographically determined CPAP setting is not always identified by patients as the most comfortable pressure setting to sustain its nightly use. When device preference is measured, more patients prefer APAP to fixed pressure CPAP $[9,23]$, although at least one study did not find any predilection for APAP [19]. While subjective ratings for sleepiness were slightly lower on APAP than fixed CPAP in some studies this finding could not be replicated in other studies $[9,18-20,21,24]$.

Clinical pearl: There may exist a marginal benefit of APAP over CPAP in terms of adherence, resolution of insomnia and residual daytime sleepiness. APAP provides a personalized approach to manage OSA in patients who have certain challenges with use of CPAP. Hence, a trial of APAP may be a reasonable treatment strategy in a carefully selected patient with history of poor adherence to CPAP due to persistent and intractable side effects.

\section{The bad}

Although overall adherence tends to be good, use of an autotitrating device does not guarantee optimal adherence to PAP therapy. Mask leak in particular is associated with poor adherence to APAP therapy [25]. Mask leak causes the pressure between the device and the upper airway to drop $[25,26]$, which can increase the residual respiratory events [25], lead to persistence or re-emergence of OSA symptoms, and result in discontinuation of therapy. Furthermore, APAP devices can underestimate the net pressure required to treat respiratory events and tend to overestimate the pressure delivered at the upper airway, which may lead to higher mask leak [26]. Optimal mask fit to preclude air leaks is of utmost importance.

Clinical pearl: Mask leak can lead to poor adherence to APAP therapy. A basic understanding of a "threshold level" of mask leak (representing maximum clinically acceptable mask leak rate) in context of the device-reported AHI can help clinicians determine whether a retitration study or mask re-fit should be the next step for a patient with persistence or re-emergence of symptoms of sleep-disordered breathing.

Some patients may experience paradoxical worsening of sleep quality with initiation of APAP therapy. In certain individuals, onset of stage rapid eye movement (REM) sleep or body position changes leading to supine posture could lead to oxygen desaturation with or without concurrent decreased airflow [15]. When these changes are sensed, the APAP device will gradually increase the pressure over next several minutes. During these transitions, when only sub-optimal pressures are provided by the APAP devices, the sustained airflow limitation or oxyhemoglobin desaturations could result in increased respiratory effort, cortical arousals or awakenings. The patient may also have difficulty acclimating to rapidly fluctuating pressures, especially if there is over-titration due to air leaks. Under these circumstances, use of APAP in a PAP-naïve patient can be particularly challenging and may compromise attempts to establish optimal titration pressure in a patient with newly diagnosed OSA [15].

Respiratory event recognition and ability to resolve detected events varies widely among different APAP devices [27]. Previous bench studies employing physiological respiratory models of upper airway collapse found that different APAP machines vary in perception and 
response when exposed to the same respiratory event (apnea, hypopnea, snoring or flow limitation) [28]. Even when two different APAP devices use a common airflow signal parameter (e.g. use of peak flow amplitude to detect hypopnea), their inherently different algorithms (e.g. $30 \%$ versus $50 \%$ decrement in peak flow amplitude) may yield significant differences in delivered pressure, which can lead to nonuniformity in reported subjective outcome parameters and adherence [29]. Most studies show overall correlation between manually scored polysomnographic AHI and automated APAP estimates of residual apnea index and AHI, especially in patients with a normal sleep efficiency (i.e., sleep efficiency $>84 \%$ ) [30-32]. Desai et al. compared the manually scored polysomnographic residual AHI to the APAPcalculated residual AHI and found that APAP devices overestimated the residual AHI at lower values and underestimated the residual AHI at higher values [32].

The tendency to underestimate residual AHI has been noted using the S9 Autoset APAP device in one study that showed the prevalence of residual sleep apnea in recruited subjects was remarkably high [33]. The tendency to overestimate AHI can also be seen at higher residual AHI values when other predisposing factors are present. One study noted the tendency to overestimate residual AHI can be present in subjects with more severe OSA at baseline, greater percentage of slow wave sleep, higher pressure requirement to abolish residual respiratory events, and in those with greater mask leak [30]. Auto-titrating devices, usually overestimate the residual hypopnea index [31,31]. APAP device reaction to repetitive hypopneas is dependent on the peak flow amplitude and inspiratory airflow shape [28], and the ability to sense these parameters depends on the algorithms utilized by each individual device. Using inspiratory flow shape instead of tidal volume as a marker for hypopnea could lead to overestimation of residual hypopnea and hence of the overall AHI [28]. Also, APAP devices may record any flow decrement as residual hypopnea, whereas manual scoring of polysomnographic data designates a hypopnea only when a flow decrement of $30 \%$ or more accompanies a minimum oxygen desaturation or cortical arousal [34]. Due to inability to distinguish sleep from wakefulness, APAP devices likely include hypopneas scored during sleep-wake transitions that would have been ignored during manual scoring of polysomnographic data $[31,33]$.

Clinical pearl: For residual respiratory events, the agreement between APAP device and manually scored polysomnographic calculations is greatest for residual apnea index, less so for residual AHI and least for residual hypopnea index.

Considerable differences exist among APAP devices with regards to sensitivity for detecting and abolishing snoring. In general, all APAP devices tend to be less sensitive in detection of snoring at high pressure settings, due to the confounding effect of increased turbine noise associated with greater turbine speed. Low intensity snoring routinely reported by bed partners can often go undetected by even the most sensitive devices [35]. Less sensitive devices and those that operate at higher pressure settings are often unable to curtail snoring [35].

Clinical pearl: APAP devices miss low intensity snoring as well as snoring that occurs when the machine delivers high pressure settings.

Although reliability in detecting residual apnea index seems good in several APAP devices, the differentiation between obstructive and central apneas is not optimal [33]. APAP devices show moderate agreement with polysomnography to identify central apneas, but unreliably low agreement for obstructive apneas [33]. The concordance between the total number of obstructive or central apneas is greater in patients with normal sleep efficiency, (i.e., sleep efficiency $>84 \%$ ) [33].
Manual scoring of polysomnographic data does not include respiratory events during wake periods. APAP devices consider the total recording time, including periods of wakefulness, to count apneas [33]. This partly explains why the overestimation of residual AHI severity can be exaggerated in patients with persistent insomnia whose obstructive sleep apnea is treated with APAP therapy.

Obstructive hypopneas, followed by central apneas, are the two most common residual respiratory events seen in patients on PAP therapy [36]. As noted previously, the tendency to overestimate obstructive hypopneas and include central apneas during wakefulness can lead to overestimation of residual AHI by auto-titrating devices. Thus, an APAP device-calculated residual AHI of $\geq 6-10$ events/hour best correlates with clinical features of residual sleep apnea and also avoids false positives [30,32]. This generalization comes with the caveat that every APAP machine has its own non-standardized algorithms for detecting respiratory events, with some newer APAP devices that claim accuracy of residual AHI estimation at values less than five [33]. This data comes from a study that defined residual sleep apnea as respiratory disturbance index (RDI) $>5$ and not $\mathrm{AHI}>5$, which in part explains the reported success of lower residual AHI on the studied APAP device as being indicative of residual OSA [33]. Given the tendency to overestimate residual respiratory events along with variable accuracy among APAP devices, high "residual AHI" figures should be interpreted in the clinical context of each patient. The decision to obtain a re-titration study or empirical pressure change based on the residual AHI reported by an APAP device should be made on a case-by-case basis.

Upper airway models that use an elastic collapsible tube (mimicking collapsible pharyngeal wall) with resistance between APAP device and the tube have compared the efficacy of different APAP devices. As APAP technology becomes more sophisticated, advancements such as "Obstructive Pressure Peak" [37] claim to better delineate obstructive from central apneas on an APAP device. Certain APAP devices use "Forced Oscillation Technology" to develop accuracy as high as standard manual titration to determine a therapeutic CPAP setting [38] However, high mask or mouth leaks may prevent adequate titration on APAP devices that monitor impedance with forced oscillation technique [15].

Clinical pearl: Given the multitude of APAP machines currently available, along with the continuous influx of newer generation APAP devices, providers need to understand device-reported values and how to use the data to individualize the care for each patient.

\section{The ugly}

Auto PAP devices tend to be more expensive than conventional CPAP devices. For newer generation APAP and CPAP devices made by the same manufacturer, average APAP device cost could be $10-50 \%$ greater than that of a conventional CPAP device. Detailed pricing for devices made by different manufacturers is listed in Table 1 [39-42] These data only represent the net prices charged by the manufacturer to the customer for private purchase, and do not reflect insurance provider purchase rates or individual copays billed to patients.

While APAP therapy enjoys a good reputation among patients and providers, its indiscriminate use in patients with obstructive sleep apnea and other medical comorbidities can make it ineffective and potentially harmful. As outlined in the 2007 American Academy of Sleep Medicine practice parameters for APAP use, patients with congestive heart failure, chronic obstructive pulmonary disease, hypoventilation syndromes, patients who do not snore (either naturally or as a result of palate 
Citation: Nigam G, Riaz M, Pathak C, Malaiya N, Shelgikar AV (2016) Use of Auto-titrating Positive Airway Pressure Devices for Sleep-disordered Breathing: The Good, the Bad and the Ugly. J Pulm Respir Med 6: 336. doi:10.4172/2161-105X.1000336

Page 4 of 6

\begin{tabular}{|c|c|c|c|c|c|c|}
\hline Manufacturer & ResMed & Philips Respironics & Fisher \& Paykel & DeVilbiss & Probasics & 3B Products \\
\hline Medicare Participation & Yes & Yes & Yes & Yes & No & No \\
\hline CPAP & $\begin{array}{c}\text { ResMed AirSense } \\
10 \text { Elite } \\
\$ 808-848\end{array}$ & $\begin{array}{c}\text { PR System One REMstar } \\
\text { Pro } \\
\$ 569-758\end{array}$ & $\begin{array}{c}\text { F\&P Icon Premo } \\
\text { CPAP } \\
\$ 489-925 \\
\end{array}$ & $\begin{array}{l}\text { DeVilbiss IntelliPAP } \\
\text { Standard Plus CPAP } \\
\quad \$ 395\end{array}$ & $\begin{array}{c}\text { Probasics Zzz-PAP } \\
\text { Auto CPAP } \\
\$ 419\end{array}$ & $\begin{array}{l}\text { RESmart CPAP } \\
\quad \$ 250-350\end{array}$ \\
\hline APAP & $\begin{array}{c}\text { ResMed AirSense } \\
\text { 10AutoSet } \\
\$ 883-923\end{array}$ & $\begin{array}{c}\text { Respironics System One } \\
\text { REMstar Auto } \\
\$ 639-828\end{array}$ & $\begin{array}{c}\text { F\&P Icon Auto } \\
\text { CPAP } \\
\$ 468-1025\end{array}$ & $\begin{array}{l}\text { DeVilbiss IntelliPAP Auto } \\
\qquad 490-545\end{array}$ & $\mathrm{~N} / \mathrm{A}$ & $\begin{array}{c}\text { RESmart Auto } \\
\text { CPAP } \\
\$ 375-470\end{array}$ \\
\hline
\end{tabular}

Table 1: Purchase Price in United States dollars (\$) for various CPAP and APAP devices as charged by third party online merchandisers ${ }^{*}$. APAP: Auto-titrating positive airway pressure; CPAP: Continuous positive airway pressure; *United States dollar figures (\$) represent prices charged to individual customer for private purchase, and do not represent copays charged by insurance providers.

surgery), and patients who have central sleep apnea syndromes are not currently candidates for APAP titration or treatment [43]. Through diligent history, physical examination, and diagnostic investigations, the primary care provider and sleep medicine specialist should exclude the above before prescription of APAP therapy. Also, clinical follow-up should ensure patients who use an APAP device have not developed any contraindications to this treatment modality.

Despite potentially similar effectiveness of APAP and CPAP to abolish residual respiratory events with APAP and CPAP treatments, conventional CPAP may be superior to APAP in reduction of cardiovascular alterations in patients with obstructive sleep apnea [44,45]. In one study systolic blood pressure, diastolic blood pressure and homeostasis model assessment index showed significant reductions in patients treated with CPAP but not in those treated with the APAP [45]. Multiple studies have shown that APAP delivers lower pressures when compared to conventional CPAP $[9,15,18]$ and the sustained high positive end expiratory pressure (PEEP) generated by conventional CPAP is potentially responsible for better oxygenation [46]. This hypothesis is well supported by one meta-analysis that showed fixed CPAP improved minimum oxygen saturation by $1.3 \%$ more than APAP therapy [20]. APAP treatment is characterized by greater sympathetic nervous system activation and a lower cardio-respiratory coupling compared with CPAP. Improved oxygenation and reduction of sympathetic tone with better cardiopulmonary coupling may promote better cardiovascular outcomes in patients treated with CPAP when compared to those treated with APAP $[20,44,45,47]$. Could high risk patients on chronic APAP therapy be at risk of worsened cardiovascular comorbidities and increased risk of long term all-cause mortality? Further long-term studies are needed to conclusively determine the effects of APAP use on cardiovascular health.

Clinical pearl: Prospective head-to-head comparison of patients treated with CPAP to those treated with APAP will help establish if the reduction in long term cardiovascular morbidity and mortality differs between the treatment modalities.

Treatment-emergent central sleep apnea could be a potential barrier to APAP adherence and efficacy. Treatment emergent central sleep apnea is a polysomnographic entity observed in some patients during positive airway pressure titration for treatment of confirmed obstructive sleep apnea. It implies emergence of central events during PAP titration after obstructive events have significantly resolved [48]. The prevalence of treatment emergent central sleep apnea varies between $5 \%$ and $20 \%$ in patients with new diagnosis of sleep-disordered breathing as found during titration study [49-56]. Treatment-emergent central sleep apnea in some cases may persist for weeks to months or may show delayed emergence [50-52,54]. Patients with higher baseline AHI, higher baseline central apnea index, advanced age, male gender and potentially those with cardiovascular comorbidities are at higher risk for development of treatment emergent central sleep apnea [4956]. Careful patient selection and follow-up is imperative to ensure no contraindications exist for patients treated with APAP, and to appropriately identify patients who require an attended titration study.

Direct prescription of APAP therapy is a missed opportunity to recognize patients who would have demonstrated treatment-emergent central sleep apneas during an attended titration study. Auto-titrating PAP machines, which tend to overestimate AHI, are not always able to distinguish closed airway from open airways apneas and are unable to distinguish central apneas of wakefulness from those in sleep. These conditions amplify the risks of over-titration, which could lead to treatment-emergent central apneas and hypopneas that in turn cause sleep disruption, poor PAP adherence and possibly worsened cardiac outcomes. Table 2 discusses some unanswered questions related to use of auto-titrating positive airway pressure therapy.

Clinical pearl: APAP titration and treatment are not recommended for use in patients with OSA with risk factors for central sleep apnea or hypoventilation syndromes. Consider an attended titration study if the patient has predisposing risk factors for primary or treatment emergent central sleep apnea.

The 2008 practice parameters for APAP use issued an option statement that "certain APAP devices may be used in an unattended way to determine a fixed CPAP treatment pressure for patients with moderate to severe OSA without significant comorbidities (congestive heart failure, chronic obstructive pulmonary disease, central sleep apnea syndromes, or hypoventilation syndromes)" [43]. So, what would be the most effective APAP setting that is representative of the "optimum" fixed CPAP treatment pressure used to treat OSA? Using a randomized control trial, Senn et al. conducted a head to head comparison of optimum pressure setting on two different APAP devices. When they used $90^{\text {th }}$ percentile of the applied pressure (in $\mathrm{cm}$ of water) from a two week adaptation period on first exposure to APAP, they found that this specific pressure setting was the same for both devices, regardless of the devices using different event algorithms to uptitrate [57]. Medians and $90^{\text {th }}$ percentiles of pressure applied by the two different devices did not statistically differ among each other. However, the $95^{\text {th }}$ percentile of applied pressures during APAP mode with either device exceeded pressures during fixed mode therapy. Gagnadoux et al. conducted an attended APAP titration and empirically used the $95^{\text {th }}$ percentile of applied pressures as the optimal pressure for fixed CPAP treatment. After three months of treatment a sleep study on this level of fixed CPAP revealed an AHI $<10$ events/hour in over $85 \%$ of their patients [58]. Given the fluctuations based on body position and sleep stages, the optimal treatment pressure may not always be the pressure setting below which $95 \%$ of all titration pressures fall (i.e., the $95^{\text {th }}$ percentile) [15].

Clinical pearl: The ideal method to determine the optimal pressure using APAP download data (i.e., 90th percentile versus $95^{\text {th }}$ percentile versus "maximum" pressure versus median pressure), still remains to be determined in most cases. As of now, the $90^{\text {th }}$ or the $95^{\text {th }}$ percentile of the applied pressure is a commonly accepted value. 
Citation: Nigam G, Riaz M, Pathak C, Malaiya N, Shelgikar AV (2016) Use of Auto-titrating Positive Airway Pressure Devices for Sleep-disordered Breathing: The Good, the Bad and the Ugly. J Pulm Respir Med 6: 336. doi:10.4172/2161-105X.1000336

Page 5 of 6

\begin{tabular}{l}
\hline \multicolumn{1}{|c|}{ Current State Question } \\
\hline $\begin{array}{c}\text { Is APAP a better treatment option than conventional CPAP during } \\
\text { pregnancy and post- partum period? }\end{array}$
\end{tabular}

\section{Conclusion}

Auto-titrating positive airway pressure offers a quick, often effective treatment for obstructive sleep apnea, though further data are needed to determine the benefit of APAP over CPAP with regards to adherence, resolution of insomnia and residual daytime sleepiness, and cardiovascular health. APAP devices show considerable interdevice variability in detection and response to respiratory events, with inconsistent ability to distinguish open airway from closed airway apneas. Data downloaded from APAP devices should always be interpreted in the context of the clinical scenario, and the clinician should use this information to determine if an attended study is necessary.

The decision to initiate or continue APAP versus CPAP should be made on a case-by case-basis, with attention to clinical and polysomnographic features, subjective barriers or benefits as reported by the patient, cost, and third party payor policies. Proper patient selection after extensive assessment of all pertinent variables will help identify patients who may gain maximal benefit from APAP therapy for treatment of obstructive sleep apnea.

\section{References}

1. AIGhanim N, Comondore VR, Fleetham J, Marra CA, Ayas NT (2008) The economic impact of obstructive sleep apnea. Lung 186: 7-12.

2. Young T, Finn L, Peppard PE, Szklo-Coxe M, Austin D, et al. (2008) Sleep disordered breathing and mortality: eighteen-year follow-up of the Wisconsin sleep cohort. Sleep 31: 1071-1078.

3. Thurnheer R, Bloch KE, Laube I, Gugger M, Heitz M, et al. (2007) Respiratory polygraphy in sleep apnoea diagnosis. Report of the Swiss respiratory polygraphy registry and systematic review of the literature. Swiss Med Wkly

\section{Future Directions}

ctive studies comparing CPAP with APAP will help determine which treatment modality is more effective in the setting of pregnancy-related hormonal and weight changes. domized controlled cross over studies comparing prevalence of central sleep apnea in treated with APAP and those with breathing to compare mortality risk. 
Citation: Nigam G, Riaz M, Pathak C, Malaiya N, Shelgikar AV (2016) Use of Auto-titrating Positive Airway Pressure Devices for Sleep-disordered Breathing: The Good, the Bad and the Ugly. J Pulm Respir Med 6: 336. doi:10.4172/2161-105X.1000336

of obstructive sleep apnea: results of a meta-analysis. Sleep 27: 249-253.

19. Vennelle M, White S, Riha RL, Mackay TW, Engleman HM, et al. (2010) Randomized controlled trial of variable-pressure versus fixed-pressure continuous positive airway pressure (CPAP) treatment for patients with obstructive sleep apnea/hypopnea syndrome (OSAHS). Sleep 33: 267-271.

20. Ip S, D'Ambrosio C, Patel K, Obadan N, Kitsios GD, et al. (2012) Auto-titrating versus fixed continuous positive airway pressure for the treatment of obstructive sleep apnea: A systematic review with meta-analyses. Syst Rev 1: 20.

21. Hukins C (2004) Comparative study of autotitrating and fixed-pressure CPAP in the home: a randomized, single-blind crossover trial. Sleep 27: 1512-1517.

22. Nolan GM, Doherty LS, Mc Nicholas WT (2007) Auto-adjusting versus fixed positive pressure therapy in mild to moderate obstructive sleep apnoea. Sleep 30: 189-194.

23. Smith I, Lasserson TJ (2009) Pressure modification for improving usage of continuous positive airway pressure machines in adults with obstructive sleep apnoea. Cochrane Database Syst Rev CD003531.

24. Noseda A, Kempenaers C, Kerkhofs M, Braun S, Linkowski P, et al. (2004) Constant vs auto-continuous positive airway pressure in patients with sleep apnea hypopnea syndrome and a high variability in pressure requirement. Chest 126: 31-37.

25. Valentin A, Subramanian S, Quan SF, Berry RB, Parthasarathy S (2011) Air leak is associated with poor adherence to autoPAP therapy. Sleep 34: 801-806.

26. Coller D, Stanley D, Parthasarathy S (2005) Effect of air leak on the performance of auto-PAP devices: a bench study. Sleep Breath 9: 167-175.

27. Schwab RJ, Badr SM, Epstein LJ, Gay PC, Gozal D, et al. (2013) An official American Thoracic Society statement: continuous positive airway pressure adherence tracking systems. The optimal monitoring strategies and outcome measures in adults. Am J Respir Crit Care Med 188: 613-620.

28. Abdenbi F, Chambille B, Escourrou P (2004) Bench testing of auto-adjusting positive airway pressure devices. Eur Respir J 24: 649-658.

29. Nolan GM, Ryan S, O'connor TM, McNicholas WT (2006) Comparison of three auto-adjusting positive pressure devices in patients with sleep apnoea. Eur Respir J 28: 159-164.

30. Ueno K, Kasai T, Brewer G, Takaya H, Maeno K, et al. (2010) Evaluation of the apnea-hypopnea index determined by the S8 auto-CPAP, a continuous positive airway pressure device, in patients with obstructive sleep apnea-hypopnea syndrome. J Clin Sleep Med 6: 146-151.

31. Ikeda Y, Kasai T, Kawana F, Kasagi S, Takaya H, et al. (2012) Comparison between the apnea-hypopnea indices determined by the REMstar auto $M$ series and those determined by standard in-laboratory polysomnography in patients with obstructive sleep apnea. Intern Med 51: 2877-2885.

32. Desai H, Patel A, Patel P, Grant BJ, Mador MJ (2009) Accuracy of autotitrating CPAP to estimate the residual Apnea-Hypopnea Index in patients with obstructive sleep apnea on treatment with autotitrating CPAP. Sleep Breath 13: $383-390$

33. Nigro CA, Gonzalez S, Arce A, Aragone MR, Nigro L (2014) Accuracy of a nove auto-CPAP device to evaluate the residual apnea-hypopnea index in patients with obstructive sleep apnea. Sleep Breath 19: 569-578.

34. Berry RB, Budhiraja R, Gottlieb DJ, Gozal D, Iber C, et al. (2012) Rules for scoring respiratory events in sleep: update of the 2007 AASM Manual for the Scoring of Sleep and Associated Events. Deliberations of the Sleep Apnea Definitions Task Force of the American Academy of Sleep Medicine. J Clin Sleep Med 8: 597-619.

35. Lofaso F, Leroux K, Quera-Salva MA, Mroue G, D'Ortho MP, et al. (2002) Snoring detection during auto-nasal continuous positive airway pressure. Eur Respir J 19: 108-112.

36. Mulgrew AT, Lawati NA, Ayas NT, Fox N, Hamilton P, et al. (2010) Residual sleep apnea on polysomnography after 3 months of CPAP therapy: clinical implications, predictors and patterns. Sleep Med 11: 119-125.

37. Ruhle KH, Domanski U, Nilius G (2013) Obstructive pressure peak: a new method for differentiation of obstructive and central apneas under auto-CPAP therapy. Sleep Breath 17: 111-115.
38. Galetke W, Randerath WJ, Stieglitz S, Laumanns C, Anduleit N, et al. (2009) Comparison of manual titration and automatic titration based on forced oscillation technique, flow and snoring in obstructive sleep apnea. Sleep Med 10: $337-343$

39. http://www.cpap.com/cpap-machines.php

40. http://www.cpapsupplyusa.com/cpap-machines/cpap-machines/machine-type/ auto.aspx

41. https://www.cpapman.com/cpap-machines/auto-cpap-machines-c-1_4.html?o sCsid=ea0a77f957ac3bec6e164d0b71e6c968

42. http://www.cpapplus.com/auto-machines

43. Morgenthaler TI, Aurora RN, Brown T, Zak R, Alessi C, et al. (2008) Practice parameters for the use of autotitrating continuous positive airway pressure devices for titrating pressures and treating adult patients with obstructive sleep apnea syndrome: An update for 2007. An American Academy of Sleep Medicine report. Sleep 31: 141-147.

44. Karasulu L, Epozturk PO, Sokucu SN, Dalar L, Altin S (2010) Improving heart rate variability in sleep apnea patients: Differences in treatment with autotitrating positive airway pressure (APAP) versus conventional CPAP. Lung 188 315-320.

45. Patruno V, Aiolfi S, Costantino G, Murgia R, Selmi C, et al. (2007) Fixed and autoadjusting continuous positive airway pressure treatments are not similar in reducing cardiovascular risk factors in patients with obstructive sleep apnea. Chest 131: 1393-1399.

46. Marini J, Wheeler A (2010) Indications and options for mechanical ventilation In: Critical Care Medicine: The Essentials ( $4^{\text {th }}$ edn.) Philadelphia: Lippincott Williams \& Wilkins p: 137.

47. Patruno V, Tobaldini E, Bianchi AM, Mendez MO, Coletti O, et al. (2014) Acute effects of autoadjusting and fixed continuous positive airway pressure treatments on cardiorespiratory coupling in obese patients with obstructive sleep apnea. Eur J Intern Med 25: 164-168.

48. Sateia MJ (2014) International classification of sleep disorders-third edition: highlights and modifications. Chest 146: 1387-1394.

49. Endo $Y$, Suzuki M, Inoue $Y$, Sato M, Namba K, et al. (2008) Prevalence of complex sleep apnea among Japanese patients with sleep apnea syndrome. Tohoku J Exp Med 215: 349-354.

50. Dernaika T, Tawk M, Nazir S, Younis W, Kinasewitz GT (2007) The significance and outcome of continuous positive airway pressure-related central sleep apnea during split-night sleep studies. Chest 132: 81-87.

51. Javaheri S, Smith J, Chung E (2009) The prevalence and natural history of complex sleep apnea. J Clin Sleep Med 5: 205-211.

52. Kuzniar TJ, Pusalavidyasagar S, Gay PC, Morgenthaler TI (2008) Natural course of complex sleep apnea--a retrospective study. Sleep Breath 12: 135139

53. Kuźniar TJ, Kasibowska-Kuźniar K, Ray DW, Freedom T (2013) Clinica heterogeneity of patients with complex sleep apnea syndrome. Sleep Breath 17: $1209-1214$.

54. Cassel W, Canisius S, Becker HF, Leistner S, Ploch T, et al. (2011) A prospective polysomnographic study on the evolution of complex sleep apnoea. Eur Respir J 38: 329-337

55. Lehman S, Antic NA, Thompson C, Catcheside PG, Mercer J, et al. (2007) Central sleep apnea on commencement of continuous positive airway pressure in patients with a primary diagnosis of obstructive sleep apnea-hypopnea. J Clin Sleep Med 3: 462-466.

56. Morgenthaler TI, Kagramanov V, Hanak V, Decker PA (2006) Complex sleep apnea syndrome: is it a unique clinical syndrome? Sleep 29: 1203-1209.

57. Senn O, Brack T, Matthews F, Russi EW, Bloch KE (2003) Randomized shortterm trial of two autoCPAP devices versus fixed continuous positive airway pressure for the treatment of sleep apnea. Am J Respir Crit Care Med 168 1506-1511.

58. Gagnadoux F, Rakotonanahary D, Martins de Araujo MT, Barros-Vieira S, Fleury B (1999) Long-term efficacy of fixed CPAP recommended by Autoset for OSAS. Sleep 22: 1095-1099. 